\title{
Clinical characteristics and biochemical mechanisms of congenital hyperinsulinism associated with dominant $\mathrm{K}_{\mathrm{ATP}}$ channel mutations
}

\author{
Sara E. Pinney, ${ }^{1}$ Courtney MacMullen, ${ }^{1}$ Susan Becker, ${ }^{1}$ Yu-Wen Lin, ${ }^{2}$ Cheryl Hanna, ${ }^{3}$
} Paul Thornton, ${ }^{4}$ Arupa Ganguly, ${ }^{5}$ Show-Ling Shyng, ${ }^{2}$ and Charles A. Stanley ${ }^{1}$

\begin{abstract}
1Division of Endocrinology/Diabetes, The Children's Hospital of Philadelphia, Philadelphia, Pennsylvania, USA.
${ }^{2}$ Center for Research on Occupational and Environmental Toxicology and ${ }^{3}$ Department of Pediatrics, Division of Endocrinology/Diabetes, Oregon Health \& Science University, Portland, Oregon, USA. “Division of Endocrinology/Diabetes, Cook Children's Medical Center, Fort Worth, Texas, USA ${ }^{5}$ Department of Genetics, University of Pennsylvania School of Medicine, Philadelphia, Pennsylvania, USA.
\end{abstract}

\begin{abstract}
Congenital hyperinsulinism is a condition of dysregulated insulin secretion often caused by inactivating mutations of the ATP-sensitive $\mathrm{K}^{+}\left(\mathrm{K}_{\mathrm{ATP}}\right)$ channel in the pancreatic $\beta$ cell. Though most disease-causing mutations of the 2 genes encoding $\mathrm{K}_{\mathrm{ATP}}$ subunits, $A B C C 8$ (SUR1) and $K C N J 11$ (Kir6.2), are recessively inherited, some cases of dominantly inherited inactivating mutations have been reported. To better understand the differences between dominantly and recessively inherited inactivating $K_{\mathrm{ATP}}$ mutations, we have identified and characterized 16 families with 14 different dominantly inherited $K_{\mathrm{ATP}}$ mutations, including a total of 33 affected individuals. The 16 probands presented with hypoglycemia at ages from birth to 3.3 years, and 15 of 16 were well controlled on diazoxide, a $K_{\mathrm{ATP}}$ channel agonist. Of 29 adults with mutations, 14 were asymptomatic. In contrast to a previous report of increased diabetes risk in dominant $K_{A T P}$ hyperinsulinism, only 4 of 29 adults had diabetes. Unlike recessive mutations, dominantly inherited $K_{\mathrm{ATP}}$ mutant subunits trafficked normally to the plasma membrane when expressed in COSm6 cells. Dominant mutations also resulted in different channel-gating defects, as dominant $A B C C 8$ mutations diminished channel responses to magnesium adenosine diphosphate or diazoxide, while dominant KCNJ11 mutations impaired channel opening, even in the absence of nucleotides. These data highlight distinctive features of dominant $\mathrm{K}_{\mathrm{ATP}}$ hyperinsulinism relative to the more common and more severe recessive form, including retention of normal subunit trafficking, impaired channel activity, and a milder hypoglycemia phenotype that may escape detection in infancy and is often responsive to diazoxide medical therapy, without the need for surgical pancreatectomy.
\end{abstract}

\section{Introduction}

Congenital hyperinsulinism comprises a group of genetic disorders of insulin secretion that cause persistent hypoglycemia in children (1). The most common and most severe of these genetic defects is associated with recessive inactivating mutations of the $\beta$ cell plasma membrane ATP-sensitive $\mathrm{K}^{+}\left(\mathrm{K}_{\mathrm{ATP}}\right)$ channel. The $\mathrm{K}_{\text {ATP }}$ channel mediates the triggering pathway of insulin secretion in which elevated levels of circulating fuels such as glucose raise the ATP/ADP ratio. The increased ATP/ADP ratio inhibits $\mathrm{K}_{\mathrm{ATP}}$ channel activity, leading to increased cytosolic calcium and release of insulin from storage granules. Disease-causing recessive $\mathrm{K}_{\text {ATP }}$ channel mutations occur in either of the 2 subunits that form the channel heterooctamer: the sulfonylurea receptor 1 (SUR1) or its regulated ion pore (Kir6.2) (2-5). These 2 protein subunits are encoded by adjacent genes, $A B C C 8$ and KCNJ11, on chromosome 11p. Recessive mutations of the $\mathrm{K}_{\mathrm{ATP}}$ channel resulting in dysregulated insulin secretion are associated with diffuse disease of the pancreatic islets when both alleles are involved, but can also cause focal $\beta$ cell adenomatosis when a paternallyderived $\mathrm{K}_{\mathrm{ATP}}$ mutation becomes expressed through embryonic loss

Nonstandard abbreviations used: AIR, acute insulin response; $\mathrm{K}_{\mathrm{ATP}}$, ATP-sensitive $\mathrm{K}^{+}$; MgADP, magnesium adenosine diphosphate.

Conflict of interest: The authors have declared that no conflict of interest exists. Citation for this article: J. Clin. Invest. 118:2877-2886 (2008). doi:10.1172/JCI35414. of heterozygosity for the maternal allele in a clone of $\beta$ cells $(6,7)$. Children with diffuse or focal disease due to recessive $\mathrm{K}_{\mathrm{ATP}}$ mutations usually present in the neonatal period with severe hypoglycemia that cannot be controlled by treatment with diazoxide or octreotide. Diazoxide, a benzothiadiazine, is a potassium channel agonist that suppresses insulin secretion in some of the forms of congenital hyperinsulinism by stimulating $\mathrm{K}_{\mathrm{ATP}}$ channel opening leading to hyperpolarization of the $\beta$ cell membrane and inhibiting the secretion of insulin. It is generally ineffective in $\mathrm{K}_{\mathrm{ATP}}$ hyperinsulinism since the $\mathrm{K}_{\mathrm{ATP}}$ channels are absent. Octreotide is a somatostatin analog that acts to suppress insulin release downstream of the $\mathrm{K}_{\text {ATP }}$ channel. It is also rarely effective in achieving long-term control of hypoglycemia possibly due to a internalization of the somatostatin receptor leading to desensitization to the drug. Because of the high risk of seizures or permanent brain damage due to hypoglycemia, children with recessive $\mathrm{K}_{\mathrm{ATP}}$ hyperinsulinism who do not respond to medical therapy frequently require near total pancreatectomy.

Recently, a few cases have been reported with mutations of the $\mathrm{K}_{\mathrm{ATP}}$ channel associated with dominantly inherited hyperinsulinism (8-12). Three of these cases had ABCC 8 mutations, and 1 had a KCNJ11 mutation. Children with these dominant $\mathrm{K}_{\mathrm{ATP}}$ hyperinsulinism mutations seemed to have a milder hypoglycemia phenotype than that seen in children with hyperinsulinism resulting from recessive $\mathrm{K}_{\mathrm{ATP}}$ mutations. Most cases appeared to be responsive to treatment 

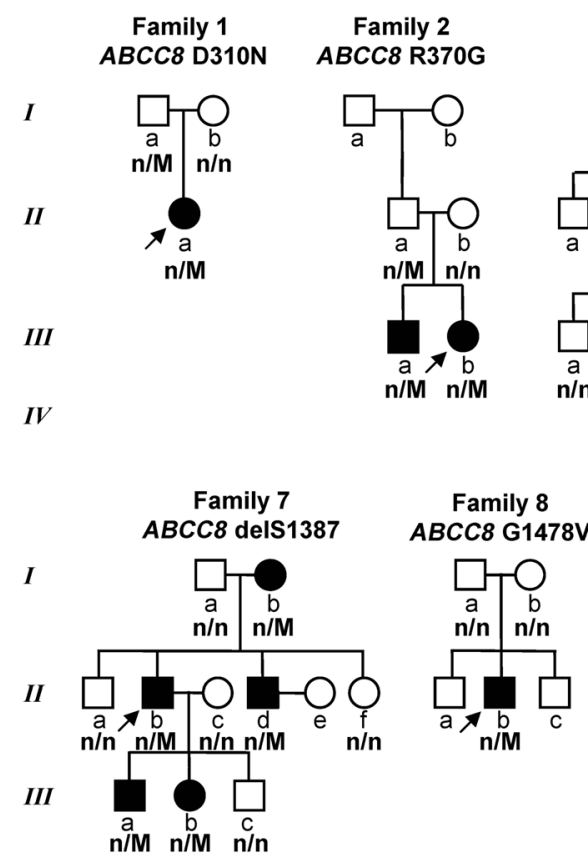

IV

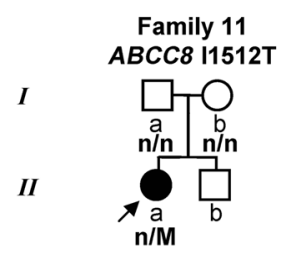

III
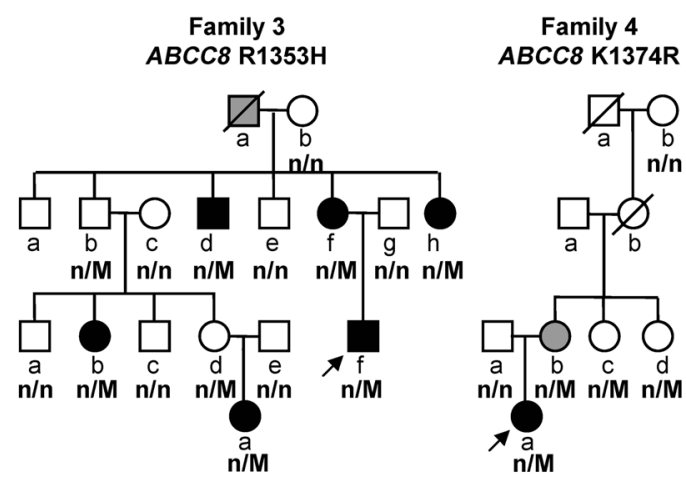

Family 6

ABCC8 delS1387

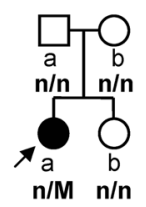

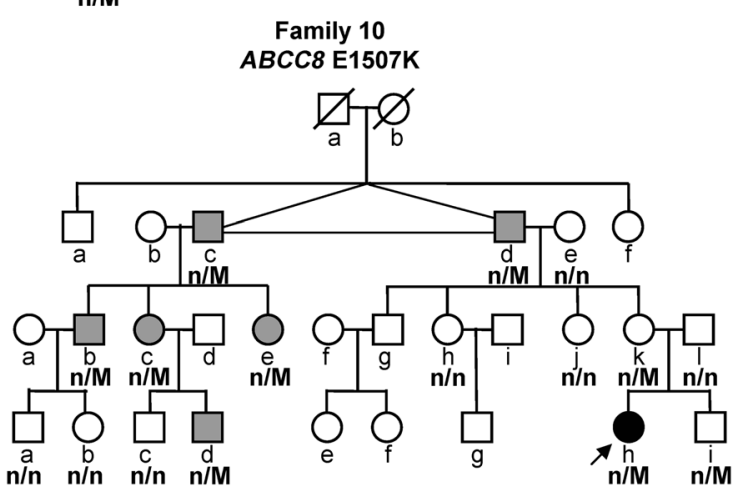

Family 14

Family 13 ABCC8 R1539Q

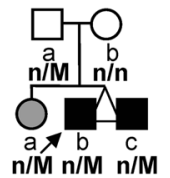

KCNJ11 F55L
Family 16 KCNJ11 D204E

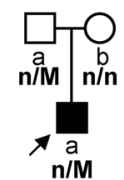

ABCC8 G1479R

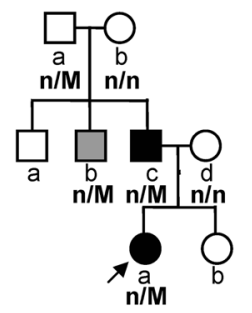

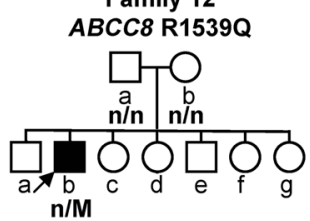

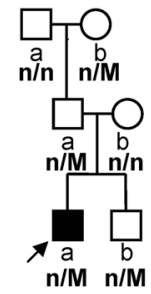

Figure 1

Pedigrees of 16 children with dominant hyperinsulinism associated with $\mathrm{K}_{\text {ATP }}$ channel mutations. The pedigrees are labeled in ascending order of SUR1 and Kir6. 2 codons. Males are depicted as squares, females as circles, and a stillborn fetus as a diamond. Identical twins are indicated by squares connected by a triangle. Arrows indicate probands. Deceased individuals are depicted with a slash. $\mathrm{n} / \mathrm{M}, \mathrm{mutation}$ positive; $\mathrm{n} / \mathrm{n}, \mathrm{mutation}$ negative; filled squares, hypoglycemia diagnosed; gray squares, hypoglycemia suspected; open squares, asymptomatic.

with diazoxide, suggesting a greater degree of residual channel function. In addition, in one of the families with a dominant inactivating $A B C C 8$ mutation, Huopio et al. suggested that the chronic elevations of cytosolic calcium concentrations led to $\beta$ cell apoptosis and an increased risk of diabetes later in life $(8,9)$. In the other 3 reported families, this high risk of diabetes was not seen. Because of the limited number of reported cases, the clinical manifestations of dominant inactivating $\mathrm{K}_{\mathrm{ATP}}$ mutations remain unclear, including responsiveness to medical therapy and later risk of diabetes. The purpose of the present study is to characterize the clinical features of dominant inactivating $\mathrm{K}_{\mathrm{ATP}}$ mutations in a large series of 16 families with hyperinsulinism and the biochemical mechanisms underlying the differences in phenotypes between dominant and recessive channel defects.

\section{Results}

Case study. The proband (Figure 1; Family 10, IVh) was the first individual in her family suspected to have hypoglycemia. She was born at term with a large-for-gestational-age birth weight of 4,063 g.
Symptomatic hypoglycemia was documented at 4 days of life and required treatment with intravenous dextrose during the first week of life. The proband was discharged from the nursery on no treatment because the large birth weight and hypoglycemia were presumed to be secondary to maternal gestational diabetes. Between 11 and 17 months of age, the infant suffered several episodes of seizures, eye deviation, and lethargy, some of which were presumed to be febrile seizures. At 17 months, she was hospitalized in status epilepticus after several days of poor feeding associated with an intercurrent illness. Hypoglycemia eventually was identified as the cause of the seizure and further investigation led to a diagnosis of hyperinsulinism. She was treated with oral diazoxide, $10 \mathrm{mg} / \mathrm{kg} / \mathrm{d}$, which resulted in good control of the hypoglycemia within 4-5 days. After starting diazoxide treatment, no further seizures occurred. The proband is now 6 years old with normal growth and development and continues to have good glycemic control on treatment with diazoxide. Genetic mutation analysis identified a single E1507K mutation in $A B C C 8$, which had been previously 
SUR1

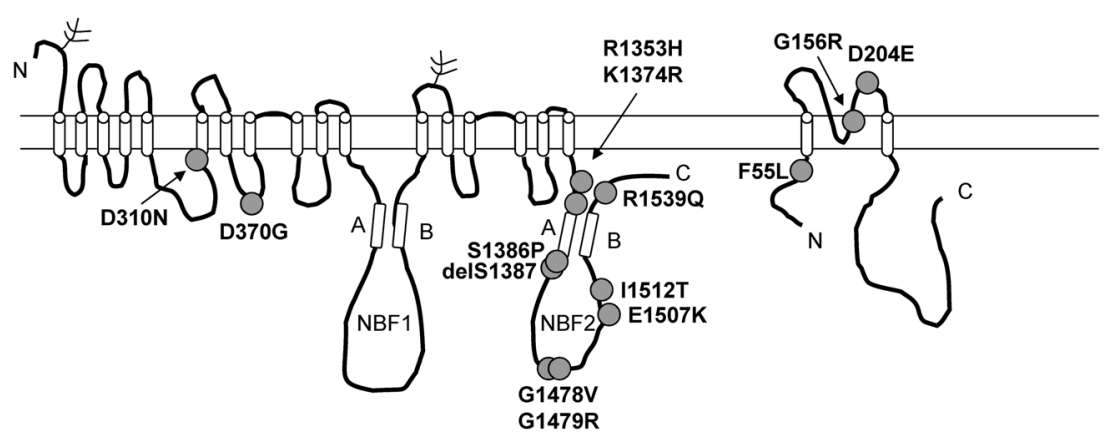

Figure 2

Locations of dominant $\mathrm{K}_{\mathrm{ATP}}$ channel mutations. Secondary structures of SUR1 and Kir6.2 are shown, including the amino $(\mathrm{N})$ and carboxy (C) termini of the proteins, SUR1 glycosylation sites, and SUR1 nuclear binding fold 1 (NBF1) and NBF2, which contain Walker A and B motifs $(A$ and $B$ ) associated with regulatory nucleotide binding. SUR1 mutations are clustered predominantly in NBF2. reported to be associated with dominant hyperinsulinism in a Finnish family by Huopio et al. $(8,9)$. The proband's family is of Eastern European origin but has no known relationship with the family described by Huopio et al.

As shown in Figure 1, 8 other members of the proband's family have been found to carry the E1507K mutation. None had been suspected to have hypoglycemia, although 3 have severe symptoms and 3 have mild symptoms consistent with hypoglycemia. The proband's mother and younger brother are the only mutation carriers who deny ever having hypoglycemic symptoms. The proband's maternal grandfather and his twin brother are both mutation carriers and have similar histories of frequent severe symptoms consistent with hypoglycemia from early childhood until their present age of 65 years. These episodes included seizure-like spells and episodes of lethargy, confusion, somnolence, or irritability, sometimes clearly provoked by fasting. The 2 brothers recall that, as schoolboys, the spells were often interpreted as inattention for which they were frequently disciplined. Patient IIIe, the daughter of the proband's great-uncle, also has a history of severe episodes of seizures. These seizures have responded poorly to anti-seizure drugs and to neurosurgical ablation therapy; and are probably indicative of severe hypoglycemia. She currently has none of the common symptoms of hypoglycemia. The older sister of IIIe, patient IIIc and her carrier son (IVd) have recurrent but milder symptoms of hypoglycemia. Patient IIIb, the brother of IIIe, has minimal symptoms of hypoglycemia with occasional irritability before meals. Two of the women in Family 10 who carry the E1507K mutation had gestational diabetes during pregnancy. One of these (IIIc) later developed obesity and diabetes requiring treatment with low doses of insulin; the second (IIIk) has not developed diabetes. The other 3 adults who carry the $\mathrm{E} 1507 \mathrm{~K}$ mutation do not have diabetes.

Figure 1 shows the pedigrees of the 16 families with dominant $\mathrm{K}_{\mathrm{ATP}}$ hyperinsulinism, all with genetic mutations found in 1 of the $2 \mathrm{~K}_{\text {ATP }}$ channel genes, $A B C C 8$ and KCNJ11. Three of these families have been reported previously (10-12), and the mutation identified in Family 10 was previously identified in an unrelated family by Huopio et al. $(8,9)$. There were a total of 14 different mutations among the 16 families. These mutations were not present in the 50 control individuals screened to determine whether these changes were common polymorphisms. All of the mutations were single amino acid changes: 13 were amino acid substitutions and 1 was a single amino acid deletion. As shown in Figure 2, the mutations were located throughout the 2 SUR1 and Kir6. 2 channel protein subunits. Interestingly, 9 of the 11 ABCC 8 mutations were concentrated in the nuclear binding fold 2 (NBF2) nucleotide-binding region of SUR1. In 12 of the 16 families, there was a

Table 1

Proband clinical data

\begin{tabular}{|c|c|c|c|c|c|c|}
\hline Family & Patient ${ }^{A}$ & $\begin{array}{l}\text { Birth weight } \\
\text { LGA }\end{array}$ & $\begin{array}{c}\text { Age of } \\
\text { presentation }\end{array}$ & $\begin{array}{l}\text { Diazoxide } \\
\text { responsive }\end{array}$ & $\begin{array}{l}\text { Current age } \\
\text { (yr) }\end{array}$ & $\begin{array}{l}\text { Additional family members affected } \\
\text { (carriers/suspected) }\end{array}$ \\
\hline 1 & Ila & Yes & $6 \mathrm{mo}$ & Yes & 10 & $1 / 0$ \\
\hline 2 & Ilb & No & $7 d$ & Yes & 5 & $2 / 0$ \\
\hline 3 & IIIf & Yes & $1 \mathrm{mo}$ & Yes & 11 & $7 / 1$ \\
\hline 4 & IVa & No & $2 \mathrm{mo}$ & Yes & 4 & $3 / 0$ \\
\hline 5 & Ila & Yes & Birth & Yes & 2 & $1 / 0$ \\
\hline 6 & Ila & Yes & $8 d$ & Yes & 9 & $0 / 0$ \\
\hline 7 & Ilb & Yes & $5 \mathrm{mo}$ & Yes & 44 & $4 / 0$ \\
\hline 8 & Ilb & Yes & $3 \mathrm{yr}, 4 \mathrm{mo}$ & Yes & 4 & $0 / 0$ \\
\hline 9 & Illa & Yes & $3 \mathrm{yr}, 1 \mathrm{mo}$ & Yes & 4 & $3 / 0$ \\
\hline 10 & IVh & Yes & $1 \mathrm{yr}, 1 \mathrm{mo}$ & Yes & 6 & $8 / 0$ \\
\hline 11 & Ila & Yes & Birth & Yes & 10 & $0 / 0$ \\
\hline 12 & Ilb & Yes & Birth & Yes & 7 & $0 / 0$ \\
\hline 13 & llb & No & Birth & Yes & 1.5 & $3 / 0$ \\
\hline 14 & Illa & No & Birth & Yes & 7 & $3 / 0$ \\
\hline 15 & Ilb & Yes & $4 \mathrm{mo}$ & No & 5 & $1 / 2$ \\
\hline 16 & Ila & Yes & Birth & Yes & 12 & $1 / 0$ \\
\hline
\end{tabular}

APatient identification as shown in Figure 1. LGA, large for gestational age. 
Table 2

Mutation carrier adults

\begin{tabular}{|c|c|c|c|c|c|}
\hline Family & Patient & $\begin{array}{l}\text { Current age } \\
\text { (yr) }\end{array}$ & $\begin{array}{l}\text { Current symptoms } \\
\text { of hypoglycemia }\end{array}$ & $\begin{array}{l}\text { Currently on } \\
\text { diazoxide }\end{array}$ & $\begin{array}{l}\text { Diagnosed } \\
\text { with diabetes }\end{array}$ \\
\hline 1 & la & 52 & No & No & No \\
\hline 2 & Ila & 32 & No & No & No \\
\hline \multirow[t]{6}{*}{3} & IIb & 51 & No & No & NIDDM \\
\hline & Ild & 49 & Yes & No & No \\
\hline & Ilf & 45 & Yes & No & GDM \\
\hline & IIh & 40 & Yes & No & No \\
\hline & IIIb & 28 & Yes & Yes & No \\
\hline & IIId & 25 & No & No & No \\
\hline \multirow[t]{3}{*}{4} & IIIb & 34 & Yes & No & No \\
\hline & IIIC & 33 & No & No & No \\
\hline & IIId & 29 & No & No & No \\
\hline 5 & lb & 32 & Yes & No & No \\
\hline \multirow[t]{3}{*}{7} & Ib & 68 & Yes & No & No \\
\hline & Ilb & 44 & No & No & NIDDMA \\
\hline & IId & 43 & Yes & Yes & No \\
\hline \multirow[t]{3}{*}{9} & la & 83 & No & No & NIDDM \\
\hline & IIb & 41 & Yes & No & No \\
\hline & IIC & 39 & Yes & No & No \\
\hline \multirow[t]{6}{*}{10} & IIC & 65 & Yes & No & No \\
\hline & Ild & 65 & Yes & No & No \\
\hline & IIIb & 45 & Yes & No & No \\
\hline & IIIC & 44 & Yes & No & NIDDM \\
\hline & Ille & 43 & No & No & No \\
\hline & IIIk & 34 & No & No & GDM \\
\hline 13 & la & 36 & No & No & No \\
\hline \multirow[t]{2}{*}{14} & $\mathrm{Ib}$ & 72 & No & No & No \\
\hline & Ila & 47 & No & No & No \\
\hline 15 & la & 34 & Yes & No & No \\
\hline 16 & la & 34 & No & No & No \\
\hline
\end{tabular}

APancreatectomy as a child. GDM, gestational diabetes.

dominant pattern of inheritance to indicate that the mutations were disease causing. In the remaining 4 families, the probands appeared to have de novo mutations (Families 6, 8, 11, and 12). Two of the de novo mutations (ABCC 8 delS1387 in Family 6 and $A B C C 8$ R1539Q in Family 12) are known to be disease causing, since they also occurred in other hyperinsulinism pedigrees, displaying dominant patterns of inheritance. The remaining 2 de novo mutations ( $A B C C 8 \mathrm{G} 1478 \mathrm{~V}$ and $\mathrm{I} 1512 \mathrm{~T}$ ) are presumed to be disease causing based on the affected probands having phenotypes similar to other probands shown in Figure 1 and results of expression studies described later.

Table 1 provides data on the phenotypes of the 16 probands in Figure 1. As illustrated in the Case study, despite frequent signs and symptoms of hypoglycemia from birth onward, the diagnosis of hyperinsulinism was delayed in the majority of the probands. Twelve of the probands (75\%) displayed manifestations of hyperinsulinism in utero with large-for-gestational-age birth weights. Only 6 (37.5\%) were diagnosed at birth, and by the end of the first month of life only a total of $8(50 \%)$ were diagnosed with hyperinsulinism. Three other infants had signs of hypoglycemia noted at birth but were not recognized to have hyperinsulinism until as late as 3 years of age.

As shown in Table 1 and Figure 1, including the 16 probands, a total of 56 individuals were identified as being affected because they carried the same mutation as a proband ( 37 cases) or had symptoms of hypoglycemia within a family of known mutation carriers (Family 3, Ia; Family 15, IIa and IIc). As illustrated in the Case study, the age of recognition of disease in these individuals and their hypoglycemia manifestations varied greatly. Some of the affected infants with overt symptoms of hypoglycemia were diagnosed at birth, while other affected family members $(n=14)$ have no reported hypoglycemia symptoms up to late adulthood, even after genetic mutation analysis was performed (as characterized in Figure 1 by open circles or squares with mutation carriers). The 16 families also include a number of symptomatic adults who were only diagnosed with hypoglycemia after the proband in the family was diagnosed with hyperinsulinism (as characterized in Figure 1 by adult mutation carriers with filled circles or squares; $n=6$ ). Table 2 shows the clinical features of the 29 mutation carriers who were greater than 18 years of age (mean 44.4 years, range $25-83$ years). Fifteen of the adults $(51.7 \%)$ have had persistent symptoms of hypoglycemia up to 68 years of age; 2 are being treated with diazoxide to control hypoglycemia. Fourteen of the adult mutation carriers do not complain of hypoglycemic symptoms similar to the mother of the proband in the Case study.

Fourteen of the 16 probands responded well to oral treatment with the $\mathrm{K}_{\mathrm{ATP}}$ channel agonist, diazoxide, with good control of hypoglycemia at doses of $5-10 \mathrm{mg} / \mathrm{kg} / \mathrm{d}$. In the other 2 , responsiveness to diazoxide was unclear. One recently diagnosed proband (Family 5, patient IIa) had been treated with a combination of diazoxide plus octreotide; his control on diazoxide alone had not been evaluated. A second proband (Family 15, patient IIb) received a pancreatectomy after treatment with diazoxide appeared not to control hypoglycemia; however, it is likely that the period of treatment, 36 hours, was too brief to judge treatment effectiveness, since the drug half-life is $24-36$ hours. Subsequently, the clinically affected younger sister of this proband responded well to treatment with diazoxide and did not require surgery. Diazoxide was effective in all of the other individuals other than probands shown in Figure 1 who required treatment to control hypoglycemic symptoms. Surgical pancreatectomy was performed in only 1 other affected individual shown in Figure 1 (Family 7, IIb). He was treated before diazoxide therapy had become available and subsequently showed a good response to treatment with diazoxide. In Family 7, patient IIIb required treatment with diazoxide as an infant but had normal fasting glucose control without therapy at age 9 . Her affected older brother (IIIa) never required diazoxide despite multiple attempts to identify fasting hypoglycemia. Withdrawal of diazoxide was not successful in some cases and, as noted in Table 2, some affected adults continued to have symptoms and require treatment. 
Table 3

Acute insulin response and oral protein tolerance tests

\begin{tabular}{|c|c|c|c|c|c|c|}
\hline Family & Patient & $\begin{array}{c}\text { Calcium } \\
\text { (AIR, } \mu \mathrm{U} / \mathrm{ml})\end{array}$ & $\begin{array}{c}\text { Leucine } \\
\text { (AIR, } \mu \mathrm{U} / \mathrm{ml})\end{array}$ & $\begin{array}{c}\text { Glucose } \\
(\text { AIR, } \mu \mathrm{U} / \mathrm{ml})\end{array}$ & $\begin{array}{l}\text { Tolbutamide } \\
\text { (AIR, } \mu \mathrm{U} / \mathrm{ml})\end{array}$ & $\begin{array}{c}\text { Oral protein test } \\
\Delta \text { glucose (nadir), } \mathrm{mg} / \mathrm{dl}\end{array}$ \\
\hline \multirow[t]{2}{*}{1} & la & 30 & ND & ND & ND & \\
\hline & Ila & ND & ND & ND & ND & $-21(57)$ \\
\hline \multirow[t]{2}{*}{2} & Ila & ND & ND & ND & ND & $+3(75)$ \\
\hline & IIIb & 0 & 8.8 & 29 & ND & \\
\hline \multirow[t]{6}{*}{3} & Ild & 10 & 0 & 25 & ND & $-16(69)$ \\
\hline & Ilf & 5.2 & 3.0 & 22 & 10 & $-8(66)$ \\
\hline & Ilh & 14 & 14 & 43 & ND & $-17(57)$ \\
\hline & IIIb & ND & ND & ND & ND & $-16(53)$ \\
\hline & Illd & 19 & 0 & 44 & ND & $-37(64)$ \\
\hline & IIIf & 2.5 & 45 & 99 & 25 & \\
\hline 4 & IIIb & 4.1 & 1.7 & 2.6 & ND & $-26(69)$ \\
\hline \multirow[t]{4}{*}{7} & $\mathrm{lb}$ & 30 & 0 & 25 & 9.5 & $-18(41)$ \\
\hline & $\mathrm{Ilb}$ & ND & ND & -1.0 & 19 & \\
\hline & Ild & 37 & 0.6 & 26 & 2.0 & $-61(50)$ \\
\hline & IIla & ND & ND & 43 & 17 & $-65(43)$ \\
\hline 8 & $\mathrm{Ilb}$ & 2.6 & 7.9 & 18 & ND & \\
\hline 9 & IIC & 47 & 11 & 66 & ND & $-32(45)$ \\
\hline 11 & Ila & 1.1 & 19 & 24 & 6.4 & $-18(56)$ \\
\hline 12 & Ilb & 2.4 & 8 & ND & 25 & \\
\hline \multirow[t]{2}{*}{14} & Ila & 9 & 13 & 114 & 44 & $-43(63)$ \\
\hline & IIla & 36 & 5 & 25 & 16 & \\
\hline Abnormal response & & $87.5 \%$ & $40 \%$ & $18.8 \%$ & $90 \%$ & $86 \%$ \\
\hline \multicolumn{7}{|c|}{ Reference values $(95 \% \mathrm{CI})^{\mathrm{A}}$} \\
\hline Recessive $\mathrm{K}_{\text {ATP }} \mathrm{HI}(n=9)$ & & 14 to 34 & 0 to 9 & 5 to 23 & -2 to 3 & \\
\hline $\mathrm{GDH} \mathrm{HI}(n=5)$ & & -8 to 4 & 15 to 70 & 63 to 170 & 23 to 36 & \\
\hline Normal controls $(n=9)$ & & -1 to 2 & -4 to 8 & 22 to 62 & 26 to 80 & \\
\hline
\end{tabular}

${ }^{A}$ Ref. 10. GDH, glutamate dehydrogenase; HI, hyperinsulinism; ND, not done.

Table 3 shows the results of tests of insulin secretory responses in the patients with dominant $\mathrm{K}_{\mathrm{ATP}}$ hyperinsulinism. Among the 16 individuals that had acute insulin response tests, 14 of $16(87.5 \%)$ had abnormal positive responses to calcium, 6 of 15 (40\%) had abnormal positive responses to leucine, 3 of 16 (18.8\%) had subnormal responses to glucose, and 9 of 10 (90\%) had a subnormal response to tolbutamide. This pattern was similar to that seen in patients with hyperinsulinism resulting from recessive mutations of the $\mathrm{K}_{\mathrm{ATP}}$ complex. However, the latter patients had more severely impaired responses to glucose and tolbutamide stimulation than the patients with dominant $\mathrm{K}_{\mathrm{ATP}}$ hyperinsulinism. Also shown in Table 3 , oral protein tolerance tests on patients with dominant $K_{\mathrm{ATP}}$ hyperinsulinism mutations demonstrated protein-sensitive hypoglycemia in 12 of 14 individuals (86\%). This was similar to patients with recessive $\mathrm{K}_{\mathrm{ATP}}$ hyperinsulinism mutations, nearly all of whom showed marked sensitivity to protein-induced hypoglycemia.

Since it has been suggested that dominant $\mathrm{K}_{\mathrm{ATP}}$ hyperinsulinism may lead to adult diabetes through $\beta$ cell apoptosis due to elevated cytosolic calcium concentrations, we reviewed the histories of the adult mutation carriers in the 16 families in Table $2(8,9)$. As stated previously, 15 of the 29 adults had symptoms of hypoglycemia, including irritability, shakiness, lethargy, and mood swings that resolve with food. Fourteen others had no history of hypoglycemic symptoms. Only 4 of the 29 adult carriers of mutations had been diagnosed with adult onset diabetes. Three of these had NIDDM. One had a near total pancreatectomy in infancy. One had been treated with oral agents for 10 years without progres- sion of diabetes and at the time of our study had symptoms of hypoglycemia suggesting persistence of hyperinsulinism. The third was diagnosed to have diabetes at 50 years of age and had been treated with oral agents for diabetes for 43 years without progression to insulin dependence. One female mutation carrier was being treated for diabetes with a modest dose of insulin after developing obesity (see Case study). Gestational diabetes during pregnancy occurred in this woman and in only 2 other female mutation carriers. In adult mutation carriers age 40 or older (excluding Family 7, patient IIb, who was post-pancreatectomy), only 3 of $17(17.6 \%)$ had developed NIDDM. The fact that 25 of the $29(86.2 \%)$ adult mutation carriers had no history of diabetes indicates that the risk of developing diabetes in patients with dominant $\mathrm{K}_{\mathrm{ATP}}$ hyperinsulinism is quite low.

Functional analysis of mutant channels. Expression studies on several of the 14 mutations, including $A B C C 8 \mathrm{R} 1353 \mathrm{H}, \triangle \mathrm{S} 1387$, G1479R, and E1507K as well as KCNJ11 F55L, have previously been published $(8,10-13)$. For the remaining mutations, their effects on channel expression and function are unknown. We therefore performed expression studies of those mutant channels in COSm 6 cells. COSm 6 cells were chosen for heterologous expression of mutant channels, as they do not express endogenous $\mathrm{K}_{\mathrm{ATP}}$ channels, allowing for clear interpretation of mutant channel phenotypes. Mutant channel expression was assessed using western blot, immunofluorescence staining, and quantitative chemiluminescence assays. Functional properties of the channel, specifically channel response to the metabolic regula- 
A

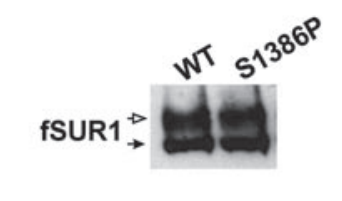

B

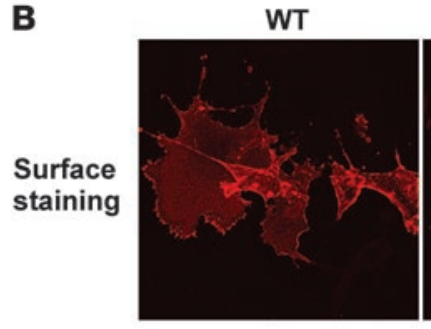

S1386P

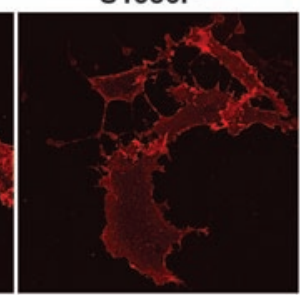

C
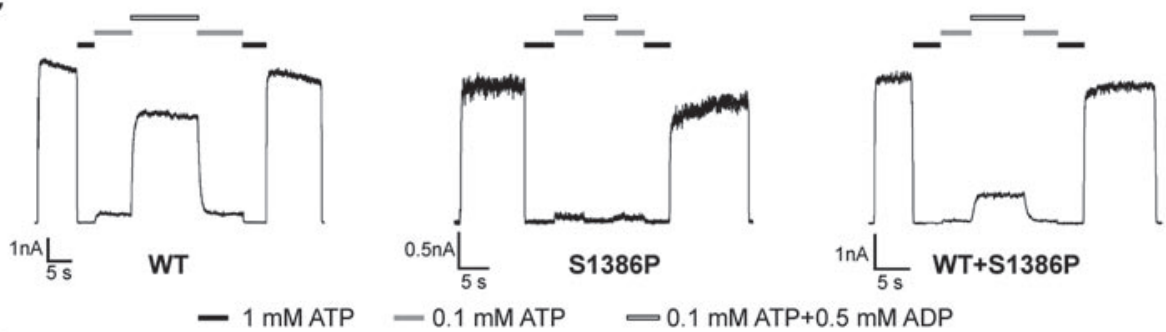

$0.1 \mathrm{mM}$ ATP $\quad \longrightarrow 0.1 \mathrm{mM} \mathrm{ATP}+0.5 \mathrm{mM}$ ADP

D
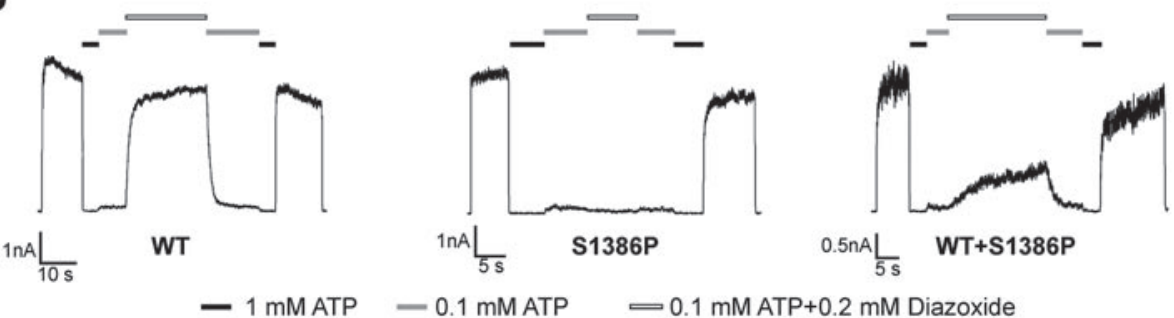

$-0.1 \mathrm{mM} \mathrm{ATP} \quad=0.1 \mathrm{mM} \mathrm{ATP}+0.2 \mathrm{mM}$ Diazoxide
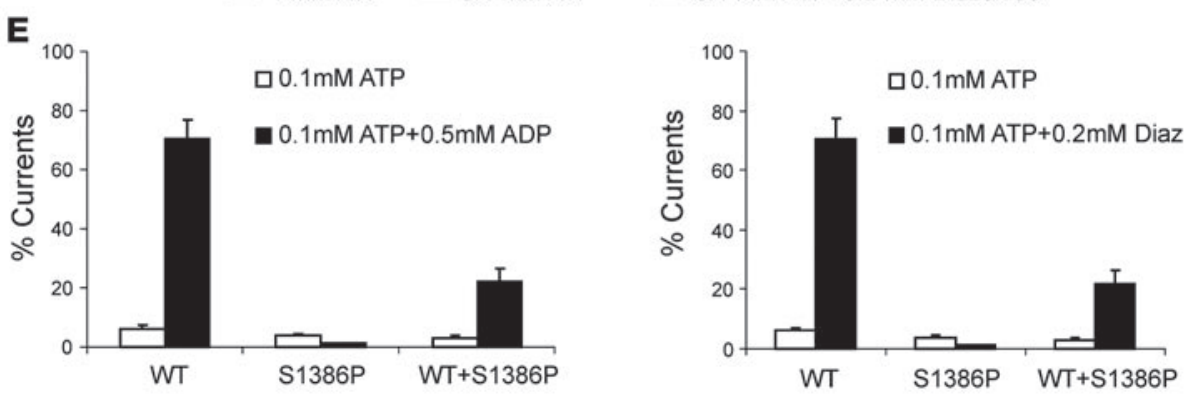

Figure 3

Analysis of mutant channels expressed in COSm6 cells. (A) Western blot analysis of fSUR1. In cells coexpressing Kir6.2 and S1386P-fSUR1, 2 bands were observed, the lower core-glycosylated band (black arrow) and the upper complex-glycosylated band (white arrow), as was seen with WT-fSUR1. (B) Confocal images of cells coexpressing Kir6.2 and either WT-fSUR1 or S1386P-fSUR1 and immunostained for surface SUR1. A and $\mathbf{B}$ illustrate that dominant $\mathrm{K}_{\mathrm{ATP}}$ mutant proteins are processed and trafficked to the membrane like the WT protein. (C and D) Representative inside-out patch-clamp recordings of WT, S1386P mutant, or WT + S1386P channels showing channel response to MgADP (C) or diazoxide (D). Currents were measured at $-50 \mathrm{mV}$ in symmetrical K-INT solution, and inward currents are shown as upward deflections. Patches were exposed to ATP, ADP, or diazoxide, as indicated by the bars above the records (the patch was also exposed to 1 mM ATP at the very beginning and end of each recording). Free $\mathrm{Mg}^{2+}$ concentration was maintained at $1 \mathrm{mM}$ in all ATP-containing solutions. Compared with WT channels, S1386P mutant channels showed a lack of response to MgADP and diazoxide, while channels from simulated heterozygous expression (WT + $\mathrm{S} 1386 \mathrm{P}$ ) showed a partial response. (E) Quantification of MgADP and diazoxide responses. Currents in $0.1 \mathrm{mM}$ ATP, $0.1 \mathrm{mM}$ ATP + $0.5 \mathrm{mM}$ ADP, or $0.1 \mathrm{mM} \mathrm{ATP}+0.2 \mathrm{mM}$ diazoxide were normalized to that seen in K-INT and expressed as percentage of currents. Each bar represents mean \pm SEM of $11(\mathrm{WT})$ or $7(\mathrm{~S} 1386 \mathrm{P}$ and $\mathrm{WT}+\mathrm{S} 1386 \mathrm{P})$ patches.

tors ATP and magnesium adenosine diphosphate (MgADP) and to the channel opener diazoxide, were evaluated by inside-out patch-clamp electrophysiological recording.

Analyses of channels harboring the S1386P-SUR1 mutation are shown as an example in Figure 3. In western blots, both WT and S1386P-fSUR1 from cells coexpressing KCNJ11 were resolved into 2 bands, an upper band that corresponds to the mature complex glycosylated form and a lower band that corresponds to the immature core glycosylated form (14). As the process leading to the complex glycosylated SUR1 occurs after correctly assembled channels have reached the medial Golgi, the intensity of the upper band is reflective of the abundance of mature channels. Figure $3 \mathrm{~A}$ shows that both upper and lower bands were of comparable intensities between the WT and mutant, suggesting that the mutation does not alter channel maturation and processing. Immunofluorescence staining of fSUR1 present at the plasma membrane demonstrated similar signal intensity between WT and mutant (Figure 3B). Quantification using the chemiluminescence assay further confirmed that the surface expres- 
Table 4

Mutant protein biogenesis and activity

\begin{tabular}{|c|c|c|c|c|c|c|}
\hline Mutant & $\begin{array}{l}\text { Surface expression, } \\
\% \text { level of WT } \\
\text { channel } \\
(n=3-5)\end{array}$ & $\begin{array}{l}\text { Average patch } \\
\text { current } \\
\text { amplitude, } n A \\
(n=7-22)\end{array}$ & $\begin{array}{l}\text { ATP sensitivity, } \\
\% \text { current in } \\
0.1 \mathrm{mM} \text { ATP } \\
(n=5-11)\end{array}$ & $\begin{array}{l}\text { MgADP stimulation, } \\
\% \text { current in } 0.1 \mathrm{mM} \\
\text { ATP + 0.5 mM ADP } \\
\quad(n=5-11)\end{array}$ & $\begin{array}{l}\text { Diazoxide stimulation, } \\
\% \text { current in } 0.1 \mathrm{mM} \\
\text { ATP + } 0.2 \mathrm{mM} \text { diazoxide } \\
(n=5-11)\end{array}$ & Ref. \\
\hline \multicolumn{7}{|l|}{ ABCC 8} \\
\hline D310N & $64.7 \pm 12.9$ & 1.6 & $5.7 \pm 2.0$ & $42.7 \pm 12.9$ & $22.8 \pm 5.6$ & \\
\hline R370G & $106.1 \pm 13.0$ & 1.9 & $3.1 \pm 0.8$ & $12.5 \pm 3.1$ & $44.1 \pm 5.8$ & \\
\hline R1353H & $89.6 \pm 6.6$ & 2.8 & $6.7 \pm 1.0$ & $45.7 \pm 4.6$ & $59.4 \pm 4.3$ & 11 \\
\hline K1374R & $126.7 \pm 7.5$ & 2.0 & $3.8 \pm 1.0$ & $10.8 \pm 3.7$ & $10.7 \pm 8.5$ & \\
\hline S1386P & $90.8 \pm 8.3$ & 2.1 & $4.0 \pm 0.5$ & $1.1 \pm 0.3$ & $2.0 \pm 0.7$ & \\
\hline DS1387 & Expressed $A$ & - & - & - & - & 10 \\
\hline G1478V & $92.5 \pm 8.0$ & 2.3 & $3.6 \pm 1.0$ & $3.5 \pm 1.0$ & $3.4 \pm 1.9$ & \\
\hline G1479R & $74.1 \pm 3.7$ & 2.2 & $7.4 \pm 1.8$ & $13.8 \pm 2.2$ & $10 \pm 3.3$ & 34 \\
\hline E1507K & $97.8 \pm 3.1$ & 3.2 & $2.6 \pm 0.8$ & $0.7 \pm 0.3$ & $3.0 \pm 0.9$ & 8,9 \\
\hline |1512T & $95.6 \pm 9.0$ & 3.6 & $5.1 \pm 1.4$ & $8.9 \pm 2.7$ & $9.1 \pm 1.4$ & \\
\hline R1539E & $72.6 \pm 10.2$ & 3.1 & $6.4 \pm 1.3$ & $12.9 \pm 4.3$ & $35.1 \pm 6.5$ & \\
\hline \multicolumn{7}{|l|}{ KCNJ11 } \\
\hline F55L & $94.4 \pm 15.6$ & 0.2 & $9.4 \pm 1.9$ & $73.9 \pm 8.0$ & $77.3 \pm 5.7$ & 12 \\
\hline G156R & $110.1 \pm 13.2$ & No current & N/A & N/A & N/A & \\
\hline D204E & $80.0 \pm 6.0$ & No current & $\mathrm{N} / \mathrm{A}$ & N/A & $\mathrm{N} / \mathrm{A}$ & \\
\hline Wild-type & 100 & 2.5 & $7.8 \pm 0.7$ & $74.8 \pm 3.7$ & $77.8 \pm 2.2$ & \\
\hline
\end{tabular}

ASurface expression normal by [125] ]iodoazidoglibenclamide photolabeling and immunofluorescence staining, but not quantified; channel activity by Rb efflux assay was severely decreased. N/A, no channel activity detected in the patches.

sion level of the S1386P mutant was not significantly different from that of WT $(90.9 \% \pm 8.3 \%$ of WT; $P>0.05 ; n=4)$ (Table 4$)$.

Having established that the mutation does not affect channel expression at the cell surface, we next determined whether the mutation affects the activity or nucleotide sensitivity of the channel by inside-out patch clamp recording. In nucleotide-free K-INT solution, the average patch current amplitude for the mutant is $2.1 \pm 0.7 \mathrm{nA}$ at $-50 \mathrm{mV}$, comparable with the $2.5 \pm 0.5 \mathrm{nA}$ observed for WT channels. Channel activities for WT and the mutant in $0.1 \mathrm{mM}$ ATP were also indistinguishable (Figure 3, C and D, and Table 4). In contrast, response to MgADP was dramatically different between WT and the mutant. In WT channels, MgADP antagonized the inhibitory effect of ATP to stimulate channel activity, whereas the mutant MgADP failed to exert such an effect (Figure 3C). We found that the mutation similarly rendered the channel unresponsive to the stimulatory effect of the $\mathrm{K}_{\mathrm{ATP}}$ channel opener diazoxide (Figure 3D). To determine how the mutation affects functional properties of the overall channel population under heterozygous state as seen in the patient, we coexpressed the WT and mutant $A B C C 8 \mathrm{cDNA}$ at 1:1 cDNA ratio together with the KCNJ11 cDNA to mimic heterozygous expression. Consistent with the clinical data, these channels exhibit partial response to MgADP and diazoxide (Figure 3, C-E). The current in $0.1 \mathrm{mM}$ ATP plus $0.5 \mathrm{mM}$ ADP relative to that observed in the absence of nucleotides was $74.8 \% \pm 3.7 \%, 22.0 \% \pm 4.4 \%$, and $1.1 \% \pm 0.3 \%$ for WT, WT + S1386P, and S1386P, respectively. The current in $0.1 \mathrm{mM}$ ATP plus $0.2 \mathrm{mM}$ diazoxide relative to that observed in the absence of nucleotides was $77.8 \% \pm 2.2 \%, 24.6 \% \pm 6.4 \%$, and $2.0 \% \pm 0.7 \%$ for WT, WT + S1386P, and S1386P, respectively.

The expression and functional data for all mutants are summarized in Table 4. The data revealed several common features shared by all $A B C C 8$ mutations. First, they had no or only mild effects on biogenesis and expression of the channel. Second, they significantly diminished or completely abolished channel response to MgADP and diazoxide. The KCNJ11 mutations also had little effect on channel trafficking and expression. However, rather than abolishing MgADP response, they either dramatically reduced the channel open probability (in the case of F55L; ref. 12) or rendered the channel nonconducting (in the cases of G156R and D204E) in the absence of nucleotides. Studies of select mutations under simulated heterozygous conditions indicated that while the mutant subunits do reduce channel function (either by reducing channel response to MgADP for $A B C C 8$ mutations or reducing channel open probability in the absence of nucleotides, in the case of KCNJ11 mutations), the WT ABCC8 or KCNJ11 allele is sufficient to confer partial channel response to changes in the ATP/ADP ratio expected during glucose metabolism and to diazoxide (an example is shown in Figure 3E).

\section{Discussion}

The results of studies in this large series of 16 families with dominant congenital hyperinsulinism caused by ABCC 8 or KCNJ11 mutations in the $\beta$ cell $\mathrm{K}_{\mathrm{ATP}}$ channel demonstrate a consistent biochemical and clinical phenotype. All of the mutations were conservative single-amino acid changes, allowing for normal channel formation at the plasma membrane. In the case of $A B C C 8$ mutations, the expressed mutant channels had impaired responsiveness to channel agonists such as diazoxide and MgADP. In the KCNJ11 mutations, the expressed channels were low conducting or nonconducting. Some individuals with dominant $\mathrm{K}_{\mathrm{ATP}}$ hyperinsulinism presented early in life with severe symptoms of hypoglycemia that cause seizures or brain injury. However, many other individuals with the mutation showed little or no evidence of hypoglycemia even with provocative testing and escaped recognition until childhood or 
later. Dominant $\mathrm{K}_{\mathrm{ATP}}$ hyperinsulinism is associated with abnormal responses to insulin secretagogues. These include a pattern of positive acute insulin responses to calcium, impaired responses to glucose and tolbutamide, as well as sensitivity to protein-induced hypoglycemia. Diazoxide is effective in controlling hypoglycemia in nearly all cases. Among the large number of adult patients described in this report, the suggestion that the risk of diabetes is increased by dominant inactivating $\mathrm{K}_{\mathrm{ATP}}$ channel mutations was not confirmed.

The hypoglycemia phenotype of dominant $\mathrm{K}_{\mathrm{ATP}}$ hyperinsulinism mutations is much milder than that of recessive $\mathrm{K}_{\mathrm{ATP}}$ hyperinsulinism mutations. Of the 39 cases with recessive $\mathrm{K}_{\mathrm{ATP}}$ hyperinsulinism we previously reported $(15,16), 100 \%$ presented within the first week of life. We have seen only 2 cases with recessive disease that presented after 6 months of age. By contrast, only $37 \%$ of the probands with dominant $\mathrm{K}_{\mathrm{ATP}}$ mutations presented before a week of age $(P<0.0001)$ and, as illustrated in the Case study, many went unrecognized until much later in life. Nevertheless, large-for-gestational-age birth weights as evidence of increased in utero insulin exposure was similar in both the recessive and dominant groups ( $87 \%$ vs. $75 \%$, respectively). It is important to note that the "milder" hypoglycemia designation in patients with dominant defects is not meant to imply that it is benign, since, as illustrated in the Case study and previous reports of Family 3 and Family 7, some of the affected individuals suffered severe symptoms of hypoglycemia, including seizures and permanent brain damage $(10,11)$.

One of the most important differences in clinical phenotype between dominant and recessive $\mathrm{K}_{\mathrm{ATP}}$ hyperinsulinism is the responsiveness to diazoxide therapy. In general, diazoxide is not effective in controlling hypoglycemia in patients with recessive disease, although partial improvements in glucose requirements are sometimes noted. In contrast, essentially all of the dominant $\mathrm{K}_{\mathrm{ATP}}$ cases in the present series achieved complete resolution of both fasting and protein-induced hypoglycemia on moderate doses of diazoxide. Although exceptions to this rule cannot be excluded as future patients are identified, complete control of hyperinsulinism by diazoxide may be a useful phenotypic marker for the dominant form of $\mathrm{K}_{\mathrm{ATP}}$ channel mutations. The difference in clinical response to diazoxide between recessive and dominant hyperinsulinism indicates that $\mathrm{K}_{\mathrm{ATP}}$ channel function is partially preserved in the dominant form. This is consistent with the observation that responses to tolbutamide and glucose appear to be impaired to a lesser degree in dominant compared with recessive $\mathrm{K}_{\mathrm{ATP}}$ hyperinsulinism patients.

Sensitivity to oral protein is an important clinical feature shared by patients with both dominant and recessive $\mathrm{K}_{\mathrm{ATP}}$ hyperinsulinism mutations. As noted previously in studies of recessive $\mathrm{K}_{\mathrm{ATP}}$ hyperinsulinism, and unlike patients with hyperinsulinism due to activating mutations of glutamate dehydrogenase, this protein sensitivity does not seem to be due to hyperresponsiveness to leucine-stimulated insulin release $(11,17)$. In vitro studies of isolated islets from SUR1-null mice confirm that the $\mathrm{K}_{\text {ATP }}$ channel defect blocks insulin response to leucine stimulation but induces a dramatic insulin response to glutamine stimulation, possibly by a direct action of this amino acid on downstream steps in insulin release when cytosolic calcium is elevated (17).

An important controversy concerning the autosomal dominant form of $\mathrm{K}_{\mathrm{ATP}}$ channel hyperinsulinism is whether the defect is a risk factor for development of adult onset diabetes. This possibility was suggested by Huopio et al. $(8,9)$ in the Finnish family with dominant hyperinsulinism due to the same $A B C C 8$ E1507K mutation found in our Family 10 (see Case study). Their proposal arose from efforts to identify susceptibility genes for type 2 diabetes and predated the recent discovery of $\mathrm{K}_{\mathrm{ATP}}$ activating mutations in permanent neonatal diabetes (18). It was based partly on observations of $\beta$ cell apoptosis in mice expressing a KCNJ11 dominant-negative transgene and on reports of $\beta$ cell apoptosis in pancreas from children with congenital hyperinsulinism $(19,20)$. In their family with the $A B C C 8$ E1507K mutations, Huopio et al. noted that 6 of 8 mothers with the mutation had been diagnosed with impaired glucose tolerance during pregnancy. They interpreted this as evidence of progressive $\beta$ cell failure, possibly due to apoptosis secondary to persistent elevation of cytosolic calcium, and thus proposed that dominant $\mathrm{K}_{\mathrm{ATP}}$ hyperinsulinism mutations were a risk factor for development of adult onset diabetes. The results in our larger group of families with dominant $\mathrm{K}_{\mathrm{ATP}}$ hyperinsulinism mutations do not support this suggestion that the defects predispose to development of diabetes. Only $13.8 \%$ of the carrier adults had been diagnosed with NIDDM and only 3 of 13 women had been diagnosed with gestational diabetes during pregnancy. The prevalence of diabetes in these adult mutation carriers is comparable with recent estimates of the prevalence of type 2 diabetes in adults in the United States, which range from $9.6 \%$ to $21 \%(21,22)$. A possible explanation for the high rate of glucose intolerance emphasized by Huopio et al. is that patients with dominant $\mathrm{K}_{\mathrm{ATP}}$ hyperinsulinism mutations have somewhat impaired insulin responses to glucose because of inadequate functioning $\mathrm{K}_{\mathrm{ATP}}$ channels (see Table 4). As noted by Remedi et al. (23), there appears to be an "inverse $U$ " curve of islet glucose responsiveness to membrane excitation, with increased insulin responses in $\mathrm{Surl}^{+/-}$or $\mathrm{Kir} 6.2^{+/-}$ mice but with decreased insulin responses to glucose in homozygous mice with complete ablation of $\mathrm{K}_{\mathrm{ATP}}$ channels. Similar impairment of insulin response to glucose stimulation has been reported by other investigators in similar knockout mouse models $(24,25)$. Similarly, insulin release in response to acute or ramp glucose stimulation is diminished in patients with recessive $\mathrm{K}_{\mathrm{ATP}}$ hyperinsulinism (26). Of note, unlike mice with dominant-negative SUR1 or Kir6.2 transgenes in which transgene expression may have induced toxicity, mice with complete ablation of either SUR1 or Kir6.2 do not show evidence of apoptosis and do not develop diabetes, indicating that $\mathrm{K}_{\mathrm{ATP}}$ channel-independent pathways of insulin regulation are capable of preventing elevations in basal glucose levels. Thus, in the case of patients with dominant or recessive $\mathrm{K}_{\mathrm{ATP}}$ inactivating mutations, impaired glucose tolerance is a direct consequence of defective $\mathrm{K}_{\mathrm{ATP}}$ activity and cannot be interpreted as a sign of impending diabetes.

The biochemical phenotype of dominant inactivating mutations of the $\mathrm{K}_{\mathrm{ATP}}$ channel also appears to be distinct from the recessive defects, which have been studied in great detail previously (27-29). The recessive mutations tend to have defects in channel biogenesis or trafficking of mature functional channels to the plasma membrane and, as a result, tend to have near absence of $\mathrm{K}_{\mathrm{ATP}}$ channel activity. Heterozygous carriers of these recessive mutations have no evidence of abnormalities in $\beta$ cell function, indicating that the mutations do not interfere with the normal allele, which is sufficient to confer normal $\beta$ cell function (26). In contrast, the dominant mutations demonstrate normal assembly with their respective WT partner and normal trafficking of assembled channels to the plasma membrane when expressed in vitro. These mutant channels when expressed as homozygous mutants in COS cells are either nonconducting or low conducting, in the case of KCNJ11 mutations, or in the case of $A B C C 8$ mutations, are incapable of opening in response to their physiological agonist $\mathrm{MgADP}$ and pharmacological agonist diazoxide. 
In heterozygous patients with these dominant $\mathrm{K}_{\mathrm{ATP}}$ mutations, the islet $\beta$ cells presumably contain an array of channel heterooctamers with 0 to 4 SUR 1 or Kir6.2 mutant subunits. In affected patients, the resulting impairment in channel activity is sufficient to activate voltage-gated calcium channels and cause abnormal insulin release, but not constitutively as occurs in patients with 2 recessive mutations. In studies simulating heterozygous expression of these dominant $K_{\text {ATP }}$ mutations (e.g., SUR1-S1386P shown in Figure 3), the overall channel response to $\mathrm{MgADP}$ or diazoxide was intermediate between that in WT and that in homomeric mutant channels. It is possible that the mutant allele has a dominant-negative effect over the WT allele and that evidence of residual functioning channels in heterozygous patients or in expression studies simulating patients represents solely the small percentage of channels that contain no mutant subunits. In the SUR1-S1386P example shown in Figure 3 , the MgADP or diazoxide response was reduced by more than $50 \%$. This would suggest that the S1386P mutation has a dominant-negative effect over the WT allele. However, it is unlikely that the dominant-negative effect is complete. In a complete dominantnegative scenario, the MgADP or diazoxide response is expected to be much less than what we observed, since only $1 / 16$ of the total channel population is expected to be pure WT channels and able to respond to MgADP and diazoxide.

Channel activities may be graded based on the number of mutant subunits present and the extent to which the mutant subunit exerts its adverse effect on channel activity in a complex containing both mutant and WT subunits. The resulting channel activity is difficult to predict and may vary depending upon the different mutations. For example, while all SUR1 mutations reduce or abolish channel response to MgADP and diazoxide without affecting channel activity in the absence of nucleotides, the 3 Kir6.2 mutations reduce channel function by preventing the pore from opening. Depending on how many mutant SUR1 or Kir6.2 subunits are involved in the functional stoichiometry of a channel, a mutant subunit may weigh in differently on the function of a channel. Future detailed characterization of these heteromeric mutant channels will be necessary to gain a full understanding of their functional impact and may shed light on the structural mechanisms regulating channel function.

In summary, these 16 families with dominant inactivating mutations of the $\mathrm{K}_{\mathrm{ATP}}$ channel genes demonstrate a distinct clinical and biochemical phenotype of congenital hyperinsulinism. The mutations associated with dominant $\mathrm{K}_{\mathrm{ATP}}$ hyperinsulinism permit normal trafficking of channel subunits to the plasma membrane, but either impair responsiveness to channel agonists such as MgADP and diazoxide or result in complete loss of channel activity. Important clinical differences from the more commonly reported recessive $\mathrm{K}_{\mathrm{ATP}}$ hyperinsulinism disorder include a milder hypoglycemia presentation and a good response to medical treatment with diazoxide so that surgical pancreatectomy is not required. Since individuals carrying dominant $\mathrm{K}_{\mathrm{ATP}}$ hyperinsulinism mutations can easily escape recognition, careful genetic and biochemical evaluation of relatives of children with medically controllable hyperinsulinism should be considered.

\section{Methods}

Patients. The 16 families described in this study represent a subgroup of patients with hyperinsulinism that were referred to and/or consulted with The Children's Hospital of Philadelphia for evaluation and treatment of congenital hyperinsulinism. Family 5 was identified and treated at the Oregon Health \& Science University, and Family 13 was diagnosed and followed at Cook Children's Medical Center (Fort Worth, Texas, USA). Patients were diagnosed with hyperinsulinism based on previously described criteria such as fasting hypoglycemia accompanied by inadequate suppression of plasma insulin, inappropriately low plasma free fatty acids and plasma $\beta$-hydroxybutyrate concentrations, and an inappropriate increase in serum glucose levels after administration of glucagon injection at the time of hypoglycemia $(30,31)$.

Acute insulin response tests. Acute insulin response (AIR) tests were carried out as previously described $(15,26,32,33)$. Patients received intravenous boluses of 4 insulin secretagogues at intervals of 60 minutes in the following sequence: calcium $(2 \mathrm{mg} / \mathrm{kg})$, leucine $(15 \mathrm{mg} / \mathrm{kg})$, glucose $(0.5 \mathrm{~g} / \mathrm{kg})$, and tolbutamide $(25 \mathrm{mg} / \mathrm{kg})$. Blood samples for insulin and glucose were obtained at $-3,-1,0,+1,+3$, and +5 minutes after the infusion of each secretagogue. Acute insulin responses were calculated as the mean increase in insulin at +1 and +3 minutes. Dextrose was infused throughout the study to maintain serum glucose levels between 60 and $80 \mathrm{mg} / \mathrm{dl}$. Criteria for abnormal positive AIR included calcium AIR $>2 \mu \mathrm{U} / \mathrm{ml}$ and leucine AIR $>8 \mu \mathrm{U} / \mathrm{ml}$. Criteria for abnormal negative AIR included glucose AIR $<22 \mu \mathrm{U} / \mathrm{ml}$ and tolbutamide $\mathrm{AIR}<26 \mu \mathrm{U} / \mathrm{ml}(10,32,34)$.

Protein tolerance tests. Oral protein tolerance tests were carried out as previously described using $1 \mathrm{~g} / \mathrm{kg}$ of protein solution (Resource Instant Beneprotein Powder, Novartis Pharmaceuticals) $(11,35)$. Abnormal response was defined as a decrease in the plasma glucose $>10 \mathrm{mg} / \mathrm{dl}$ accompanied by a glucose nadir $<70 \mathrm{mg} / \mathrm{dl}$ or an absolute decrease in plasma glucose of $15 \mathrm{mg} / \mathrm{dl}$ within 3 hours of protein ingestion.

Genetic analysis. Peripheral blood samples were obtained from patients and family members for isolation of genomic DNA (Gentra). If blood was not available, saliva was collected via the Oragene DNA Self Collection Kit (DNA Genotek) and extracted via the manufacturer's protocol.

The coding sequences of the $A B C C 8$ and $K C N J 11$ genes were amplified and directly sequenced on an ABI 3730 capillary DNA analyzer (ABI) as previously described (11). Resulting chromatograms were analyzed via the Sequencher 4.7 program (Gene Codes Corp). ABCC 8 cDNA nucleotides and amino acids were numbered according to the sequence reported by Nestorowicz et al. (3) that includes the alternatively spliced exon 17 sequence (National Center for Biotechnology Information accession no. L78224). Therefore, the E1506K mutation described in the reports by Huopio et al. $(8,9)$ is the same amino acid change as the $\mathrm{E} 1507 \mathrm{~K}$ mutation in the Case study. Once mutations were confirmed, DNA from 50 individuals participating in the DNA Polymorphism Discovery Resource (Coriell Cell Depository) was sequenced to rule out the possibility that the change was a rare polymorphism.

Functional analysis of mutant channels. Hamster SUR1 and rat Kir6.2 cDNAs were used for functional expression, as they are nearly identical to their human counterparts at the amino acid level and have been extensively employed in prior functional studies. All amino acids altered by human SUR1 and Kir6.2 mutations identified in the current study are conserved in hamster SUR1 and rat Kir6.2. Point mutations of SUR1 or Kir6.2 were introduced into hamster SUR1 cDNA in the pECE plasmid or rat Kir6.2 cDNA in PCDNAI using the QuickChange site-directed mutagenesis kit (Stratagene) as previously described (36). Mutations were confirmed by DNA sequencing. COSm6 cells were plated onto $35-\mathrm{mm}$ culture dishes and transfected with WT or mutant SUR1 and WT or mutant Kir6.2 cDNA using FuGENE 6 (Roche).

Cell surface expression of mutant channels was assessed by western blot, fluorescent staining, and a quantitative chemiluminescence assay as described previously (36). For these experiments, SUR1 tagged with a FLAG epitope at the $\mathrm{N}$ terminus was used. The FLAG epitope tag has been shown not to affect channel expression and functional properties in prior studies (28).

Functional properties of channels were studied using inside-out patchclamp recordings as previously described (36). Briefly, COSm6 cells were transfected with cDNA encoding WT or mutant channel proteins as well 
as cDNA for the GFP for identification of transfected cells. Patch-clamp recordings were made 36-72 hours after transfection. Micropipettes were pulled from nonheparinized Kimble glass (Fisher Scientific) with resistance typically $\sim 1.0-1.5$. The bath (intracellular) and pipette (extracellular) solution (K-INT) had the following composition: $140 \mathrm{mM} \mathrm{KCl}, 10 \mathrm{mM}$ K-HEPES, 1 mM K-EGTA, pH 7.3. ATP and ADP were added as the potassium salt. All currents were measured at membrane potential of $-50 \mathrm{mV}$ and inward currents shown as upward deflections. Data were analyzed using pCLAMP software (Axon Instrument). The MgADP or diazoxide response was calculated as the current in K-INT solution plus $0.1 \mathrm{mM} \mathrm{ATP}, 0.5 \mathrm{mM}$ $\mathrm{ADP}$ or $0.2 \mathrm{mM}$ diazoxide, and $1 \mathrm{mM}$ free $\mathrm{Mg}^{2+}$ relative to that in plain K-INT solution. Statistical analysis was performed using independent 2-population 2-tailed Student's $t$ test.

Written informed consent was obtained from all patients and family members for this study. This study was reviewed and approved by The Children's Hospital of Philadelphia Institutional Review Board.

\section{Acknowledgments}

The authors wish to thank the nurses and staff of The Children's Hospital of Philadelphia and Clinical Translational Research Core in caring for the patients and carrying out the testing. We thank the nurse practitioners in The Congenital Hyperinsulinism Center at The Children's Hospital of Philadelphia for their assistance in obtaining clinical information about the families. We would also thank Jillene Casey, Malini Kochhar and Joaquin Espinoza Goodman from Oregon Health \& Science University for their technical assistance. This work was supported in part by the following grants from the NIH: RO1 DK56268, RO1 DK53012, and T32 DK63688 (to C.A. Stanley), RO1 DK57699 and RO1 DK66485 (to S.-L. Shyng), and UL1 RR024134. S.E. Pinney was the recipient of fellowship awards from the Lawson Wilkins Pediatric Endocrine Society and the Institute of Translational Medicine and Therapeutics at the University of Pennsylvania School of Medicine.

Received for publication February 22, 2008, and accepted in revised form May 19, 2008.

Address correspondence to: Charles A. Stanley, Division of Endocrinology, The Children's Hospital of Philadelphia, 3516 Civic Center Boulevard, ARC 802, Philadelphia, Pennsylvania 19104, USA. Phone: (215) 590-3420; Fax: (215) 590-1605; E-mail: stanleyc@email.chop.edu.
1. De Leon, D.D., and Stanley, C.A. 2007. Mechanism of disease: advances in diagnosis and treatment of hyperinsulinism. Nat. Clin. Pract. Endocrinol. Metab. 3:57-58.

2. Nestorowicz, A., et al. 1997. A nonsense mutation in the inward rectifier potassium channel gene, Kir6.2, is associated with familial hyperinsulinism. Diabetes. 46:1743-1748.

3. Nestorowicz, A., et al. 1996. Mutations in the sulonylurea receptor gene are associated with familial hyperinsulinism in Ashkenazi Jews. Hum. Mol. Genet. 5:1813-1822.

4. Thomas, P., Ye, Y., and Lightner, E. 1996. Mutation of the pancreatic islet inward rectifier Kir6.2 also leads to familial persistent hyperinsulinemic hypoglycemia of infancy. Hum. Mol. Genet. 5:1809-1812.

5. Thomas, P.M., et al. 1995. Mutations in the sulfonylurea receptor gene in familial persistent hyperinsulinemic hypoglycemia of infancy. Science. 268:426-429.

6. de Lonlay-Debeney, P., et al. 1999. Clinical features of 52 neonates with hyperinsulinism. N. Engl. J. Med. 340:1169-1175.

7. de Lonlay, P., et al. 2005. Dominantly inherited hyperinsulinaemic hypoglycaemia. J. Inherit. Metab. Dis. 28:267-276.

8. Huopio, H., et al. 2000. Dominantly inherited hyperinsulinism caused by a mutation in the sulfonylurea receptor type 1. J. Clin. Invest. 106:897-906.

9. Huopio, H., et al. 2003. A new subtype of autosomal dominant diabetes attributable to a mutation in the gene for sulfonylurea receptor 1 . Lancet. 361:301-307.

10. Thornton, P.S., et al. 2003. Clinical and molecular characterization of a dominant form of congenital hyperinsulinism caused by a mutation in the high-affinity sulfonylurea receptor. Diabetes. 52:2403-2410.

11. Magge, S.N., et al. 2004. Familial leucine-sensitive hypoglycemia of infancy due to a dominant mutation of the beta-cell sulfonylurea receptor. J. Clin. Endocrinol. Metab. 89:4450-4456.

12. Lin, Y.W., MacMullen, C., Ganguly, A., Stanley, C.A., and Shyng, S.L. 2006. A novel KCNJ11 mutation associated with congenital hyperinsulinism reduces the intrinsic open probability of beta-cell ATP-sensitive potassium channels. J. Biol. Chem. 281:3006-3012.
13. Nichols, C.G., et al. 1996. Adenosine diphosphate as an intracellular regulator of insulin secretion. Science. 272:1785-1787.

14. Clement, J.P., 4th, et al. 1997. Association and stoichiometry of K(ATP) channel subunits. Neuron. 18:827-838.

15. Stanley, C.A., et al. 2004. Preoperative evaluation of infants with focal or diffuse congenital hyperinsulinism by intravenous acute insulin response tests and selective pancreatic arterial calcium stimulation. J. Clin. Endocrinol. Metab. 89:288-296.

16. Hardy, O.T., et al. 2007. Accuracy of [18F]fluorodopa positron emission tomography for diagnosing and localizing focal congenital hyperinsulinism. J. Clin. Endocrinol. Metab. 92:4706-4711.

17. Li, C., et al. 2004. A signaling role of glutamine in insulin secretion. J. Biol. Chem. 279:13393-13401.

18. Gloyn, A.L., et al. 2004. Activating mutations in the gene encoding the ATP-sensitive potassium-channel subunit Kir6.2 and permanent neonatal diabetes. N. Engl.J. Med. 350:1838-1849.

19. Kassem, S.A., Ariel, I., Thornton, P.S., Scheimberg, I., and Glaser, B. 2000. Beta-cell proliferation and apoptosis in the developing normal human pancreas and in hyperinsulinism of infancy. Diabetes. 49:1325-1333.

20. Miki, T., et al. 1997. Abnormalities of pancreatic islets by targeted expression of a dominant-negative KATP channel. Proc. Natl. Acad. Sci. U. S. A. 94:11969-11973.

21. Signorello, L.B., et al. 2007. Comparing diabetes prevalence between African Americans and Whites of similar socioeconomic status. Am. J. Public Health. 97:2260-2267.

22. Centers for Disease Control and Prevention. 2005. CDC Diabetes Fact Sheet: United States, 2005. http://www.cdc.gov/Diabetes/pubs/pdf/ndfs_ 2005.pdf.

23. Remedi, M.S., et al. 2006. Hyperinsulinism in mice with heterozygous loss of K(ATP) channels. Diabetologia. 49:2368-2378.

24. Seghers, V., Nakazaki, M., DeMayo, F., AguilarBryan, L., and Bryan, J. 2000. Sur1 knockout mice. A model for K(ATP) channel-independent regulation of insulin secretion. J. Biol. Chem. 275:9270-9277.

25. Shiota, C., et al. 2002. Sulfonylurea receptor type 1 knock-out mice have intact feeding-stimulated insulin secretion despite marked impairment in their response to glucose. J. Biol. Chem. 277:37176-37183.

26. Grimberg, A., et al. 2001. Dysregulation of insulin secretion in children with congenital hyperinsulinism due to sulfonylurea receptor mutations. Diabetes. 50:322-328.

27. Yan, F.F., et al. 2007. Congenital hyperinsulinism associated $\mathrm{ABCC} 8$ mutations that cause defective trafficking of ATP-sensitive K+ channels: identification and rescue. Diabetes. 56:2339-2348.

28. Cartier, E.A., Conti, L.R., Vandenberg, C.A., and Shyng, S.L. 2001. Defective trafficking and function of KATP channels caused by a sulfonylurea receptor 1 mutation associated with persistent hyperinsulinemic hypoglycemia of infancy. Proc. Natl. Acad. Sci. U. S. A. 98:2882-2887.

29. Shyng, S.L., et al. 1998. Functional analyses of novel mutation in the sulfonyurea receptor 1 with persistent hyperinsulinemic hypoglycemia of infancy. Diabetes. 47:1145-1151.

30. Finegold, D.N., Stanley, C.A., and Baker, L. 1980. Glycemic response to glucagon during fasting hypoglycemia: an aid in the diagnosis of hyperinsulinism. J. Pediatr. 96:257-259.

31. Stanley, C.A., and Baker, L. 1976. Hyperinsulinism in infancy: diagnosis by demonstration of abnormal response to fasting hypoglycemia. Pediatrics. 57:702-711.

32. Ferry, R.J., Jr., et al. 2000. Calcium-stimulated insulin secretion in diffuse and focal forms of congenital hyperinsulinism. J. Pediatr. 137:239-246.

33. Henwood, M.J., et al. 2005. Genotype-phenotype correlations in children with congenital hyperinsulinism due to recessive mutations of the adenosine triphosphate-sensitive potassium channel genes. J. Clin. Endocrinol. Metab. 90:789-794.

34. Kelly, A., et al. 2001. Acute insulin responses to leucine in children with the hyperinsulinism/hyperammonemia syndrome. J. Clin. Endocrinol. Metab. 86:3724-3728.

35. Hsu, B.Y., et al. 2001. Protein-sensitive and fasting hypoglycemia in children with the hyperinsulinism/hyperammonemia syndrome. J. Pediatr. 138:383-389.

36. Taschenberger, G., et al. 2002. Identification of a familial hyperinsulinism-causing mutation in the sulfonylurea receptor 1 that prevents normal trafficking and function of KATP channels. J. Biol. Chem. 277:17139-17146. 